\title{
miR-331 inhibits CLDN2 expression and may alleviate the vascular endothelial injury induced by sepsis
}

\author{
LINGCHEN KONG, PENG WU and JIANZHONG LI \\ Department of Critical Care Medicine, Linyi Central Hospital, Linyi, Shandong 276400, P.R. China
}

Received October 15, 2017; Accepted March 11, 2020

DOI: $10.3892 / \mathrm{etm} .2020 .8854$

\begin{abstract}
The present study aimed to determine the expression level of claudin-2 (CLDN2) in the peripheral blood of patients with sepsis, and to investigate its potential function and mechanism of action in vascular endothelial injury. A total of 25 patients with sepsis were included in the present study. Reverse transcription-quantitative PCR was used to determine CLDN2 levels in peripheral blood. HUVECs stably expressing CLDN2 were prepared and Cell Counting Kit-8, flow cytometry and Transwell assays were performed to study the proliferation, apoptosis and migration of HUVECs, respectively. Using bioinformatics, microRNA (miR) molecules that interact with CLDN2 were predicted. A dual luciferase reporter assay was used to test whether miR-331 regulated CLDN2. Western blotting was employed to determine CLDN2 protein expression. In addition, in vitro transfection of HUVECs with miR-331 mimics was performed to test the rescue effects of miR-331 on the cell function changes induced by CLDN2. The results indicated that elevated CLDN2 expression altered the proliferation and cell cycle of peripheral vascular endothelial cells. CLDN2 overexpression inhibited HUVEC proliferation via mechanisms not associated with the cell cycle. CLDN2 mRNA levels in the peripheral blood of patients with sepsis were significantly higher than those in healthy subjects. Upregulated CLDN2 expression promoted the apoptosis of HUVECs, but reduced their proliferation and migration. Notably, miR-331 was able to bind with CLDN2 mRNA and regulate its expression. Upregulation of miR-331 expression inhibited the expression of CLDN2 and restored nearly normal proliferation, apoptosis and migration to HUVECs. The present study demonstrated that CLDN2 expression is elevated in peripheral blood from patients with sepsis, and promotes the injury of vascular endothelial cells. In addition, miR-331 participates in the direct regulation of CLDN2, and
\end{abstract}

Correspondence to: Dr Jianzhong Li, Department of Critical Care Medicine, Linyi Central Hospital, 17 Jiankang Road, Linyi, Shandong 276400, P.R. China

E-mail: lwgs321@163.com

Key words: miR-331, claudin-2, vascular endothelial injury, sepsis upregulation of miR-331 expression inhibits the expression of CLDN2 and restores cellular functions to HUVECs.

\section{Introduction}

Sepsis is a common disease with a worldwide incidence of $10 \%$ affecting patients in intensive care units that is associated with the systemic inflammatory response syndrome caused by infection (1), and is mainly caused by pathogenic microorganisms or toxins that invade the circulatory system $(2,3)$. The clinical manifestations of sepsis include fever, shortness of breath and peripheral leukocytosis, and severe sepsis is accompanied by organ dysfunction and tissue hypoperfusion (4). Without timely treatment, it can result in septic shock, which causes acute circulatory failure or death (5). Sepsis is a dangerous condition with a mortality rate of $>40 \%$ between 1993-2003 in the United States of America (6). At present, the treatment for sepsis is mainly based on the correction of pathophysiological changes, and no effective cure is available (7).

The pathogenesis of sepsis is complicated. The release of large amounts of inflammatory mediators caused by infection initiates a cascade reaction in systemic tissues and organs, forms a complex inflammatory network and ultimately causes cell damage $(8,9)$. In addition, the occurrence and development of sepsis are closely associated with abnormal coagulation, immune dysfunction, abnormal gene expression, tissue damage and other pathophysiological changes (10). Vascular endothelial injury is one of the pathological changes occurring in sepsis and it serves an important role in mild and severe sepsis (3). When sepsis occurs, toxins and inflammatory factors in the blood cause damage to vascular endothelial cells. In addition to destruction of the vascular endothelial barrier, the damaged endothelial cells may exhibit secretory dysfunction (11). Furthermore, damaged endothelial cells continue to secrete inflammatory factors to promote an inflammatory response, leading to dysfunctional vasoconstriction and the aggravation of tissue- and microcirculation hypoperfusion $(12,13)$. Therefore, the status of vascular endothelial cells is important in the maintenance of normal vascular functions, and is an important target in the clinical treatment of sepsis.

A tight junction is a key cell structure for sustaining the vascular endothelial barrier. It is closely associated with the exchange of substances among cells, the secretion of cytokines and the regulation of signaling pathways (14). Claudin-2 (CLDN2) is a tight junction-associated protein (15). 
The claudin family currently has 24 identified members that are involved in the formation and maintenance of intercellular tight junctions (16). Abnormal expression of claudin family members is closely associated with various pathophysiological processes (17). Claudin family proteins are involved in the regulation of the occurrence and development of inflammation in tissues and organs (18). In tumors, the abnormal expression of claudin family members is associated with tumor cell proliferation, drug resistance, apoptosis and distant metastasis, and these proteins are targets in diagnosis and treatment (19). A study showed that the CLDN2 gene is associated with chronic pancreatitis, colitis and cholangitis (20). However, to date, the role and regulation of CLDN2 in vascular endothelial injury are not clear.

MicroRNA (miRNA/miR) is a class of non-coding small RNAs (18-22 nucleotides) that bind to the 3'-untranslated region (UTR) of target genes and regulate their expression (21). At present, it is not clear whether miRNAs are involved in the regulation of CLDN2 during vascular injury. In a preliminary bioinformatics analysis conducted by the present research team, it was found that CLDN2 mRNA is a potential target of miR-331. Additionally, it has been reported that miR-331 is associated with the PM2.5-induced injury of respiratory epithelial cells (22). In the present study, the function and upstream regulation mechanism of CLDN2 in vascular endothelial injury induced by sepsis and the roles of tight junction proteins in sepsis were investigated.

\section{Materials and methods}

Patients. A total of 25 patients with sepsis that received treatment at Linyi Central Hospital (Linyi, China) between December 2015 and December 2016 were included in the present study. The patients comprised 18 males and 7 females with a mean age of $48.5 \pm 5.7$ years and age range of 38-56 years. The included sepsis patients did not have any one of the exclusion criteria: History of autoimmune diseases, diabetes, hypertension and long-term medication. In addition, 20 healthy subjects (14 males and 6 females) aged between $22-45$ years (mean age, $38 \pm 4.3$ years) who undertook physical examination at the same hospital during the same recruitment time with the patients were included as a control group. Peripheral blood $(5 \mathrm{ml})$ was collected from all patients and healthy subjects. To determine CLDN2 gene and miR-331 expression, $250 \mu \mathrm{l}$ blood was used. The remaining $4.75 \mathrm{ml}$ was used for the separation of serum by centrifugation at $600 \mathrm{x} \mathrm{g}$ and $4^{\circ} \mathrm{C}$ for $2 \mathrm{~min}$. Serum samples were stored at $-80^{\circ} \mathrm{C}$. All procedures were approved by the Ethics Committee of Linyi Central Hospital. Written informed consent was obtained from all patients.

Reverse transcription-quantitative PCR (RT-qPCR). Peripheral blood $(250 \mu \mathrm{l})$ was mixed with $750 \mu \mathrm{l}$ TRIzol ${ }^{\circledR}$ reagent (Thermo Fisher Scientific, Inc.) for lysis. Then, total RNA was extracted using the phenol-chloroform method. The concentration and quality of RNA was measured using UV spectrophotometry. cDNA was obtained by RT from $1 \mu \mathrm{g}$ RNA using the TIANScript II cDNA First Strand Synthesis kit (Tiangen Biotech Co., Ltd.) and RT of miRNA was performed using miRcute miRNA cDNA First Strand Synthesis kit (Tiangen
Biotech Co., Ltd.) according to the manufacturers' protocols. BeyoFast SYBR-Green qPCR mix kit (Beyotime Institute of Biotechnology) was used to detect mRNA expression of CLDN2, using GAPDH as internal reference. The primer sequences were: CLDN2, forward, 5'-CCTTTATCACCTCAG CCCGT-3' and reverse, 5'-GCTACCGCCACTCTGTCTTT-3'; and GAPDH, forward, 5'-CGGAGTCAACGGATTTGGTCG TAT-3' and reverse, 5'-AGCCTTCTCCATGGTGGTGAA GAC-3'. The thermocycling conditions were as follows: $95^{\circ} \mathrm{C}$ for $10 \mathrm{~min}$, followed by 40 cycles of $95^{\circ} \mathrm{C}$ for $1 \mathrm{~min}$ and $60^{\circ} \mathrm{C}$ for $30 \mathrm{sec}$. The expression of miR-331 was determined by miRcute miRNA RT-PCR kit (Tiangen Biotech Co., Ltd.), using U6 as internal reference. Primer sequences were: miR-331, 5'-GCCCCUGGGCCTATCCTAGAA-3' (single strand primer); and U6, forward, 5'-CTCGCTTCGGCAGCACA-3' and reverse, 5'-AACGCTTCACGAATTTGCGT-3'. The thermocycling conditions were as follows: $95^{\circ} \mathrm{C}$ for $10 \mathrm{~min}$, followed by 40 cycles of $95^{\circ} \mathrm{C}$ for $1 \mathrm{~min}$ and $60^{\circ} \mathrm{C}$ for $30 \mathrm{sec}$. The $2^{-\Delta \Delta \mathrm{Cq}}$ method (23) was used to calculate relative expression against the internal reference. Each sample was tested in triplicate.

Cells. HUVECs (The Cell Bank of Type Culture Collection of the Chinese Academy of Sciences) were cultured in RPMI-1640 medium (BD Biosciences) supplemented with $10 \%$ FBS (BD Biosciences) at $37^{\circ} \mathrm{C}$ and $5 \% \mathrm{CO}_{2}$. A total of $2 \times 105$ cells/well were seeded in 24 -well plates and divided into negative control (NC) and sepsis groups. When reaching $70-90 \%$ confluence, $250 \mu \mathrm{l}$ serum from sepsis patients and $250 \mu 1$ RPMI-1640 with $10 \%$ FBS were added to the cells in the sepsis group, and $250 \mu \mathrm{l}$ serum from healthy subjects and $250 \mu \mathrm{l}$ RPMI-1640 with $10 \%$ FBS were added to the cells in the NC group. HUVECs were cultured for $24 \mathrm{~h}$ before subsequent tests were performed as previously described $(24,25)$.

To transfect HUVECs with miR-NC (cat. no. B04001; Shanghai GenePharma Co., Ltd.) or miR-331 mimics (5'-CTAGGTATGGTCCCAGGGATCC-3'; Shanghai GenePharma Co., Ltd.) cells $\left(2 \times 10^{5}\right)$ were seeded in $24-$ well plates and cultured in RPMI-1640 with $10 \%$ FBS until $70 \%$ confluence was reached. In the first vial, $1.5 \mu \mathrm{lmiR}-331 \mathrm{mimic}$ (20 pmol/ $\mu \mathrm{l}$; Hanbio Biotechnology Co., Ltd.) was mixed with $50 \mu 1$ Opti-MEM (Thermo Fisher Scientific, Inc.). In the second vial, $1 \mu$ l Lipofectamine 2000 (Thermo Fisher Scientific, Inc.) was mixed with $50 \mu \mathrm{l}$ Opti-MEM. After incubation at room temperature for $5 \mathrm{~min}$, the two vials were combined and incubated at room temperature for $20 \mathrm{~min}$. Then, the mixtures were added to cells in the respective groups. Six hours later, the medium was replaced with RPMI-1640 with 10\% FBS. After cultivation for $48 \mathrm{~h}$, cells were collected for further assays.

For infection with Lv-puro-CLDN2 overexpression vector, HUVECs $\left(1 \times 10^{5}\right)$ were seeded in 24-well plates and cultured until $70 \%$ confluence was reached. Then, Lv-puro-NC and Lv-puro-CLDN2 lentiviral vectors were added to the cells (multiplicity of infection, 20; Hanbio Biotechnology Co., Ltd.). After incubation at $37^{\circ} \mathrm{C}$ with $5 \% \mathrm{CO}_{2}$ for $12 \mathrm{~h}$, the medium was replaced with RPMI-1640 containing $1 \mu \mathrm{g} / \mathrm{ml}$ puromycin (cat. no. A1113803; Gibco; Thermo Fisher Scientific, Inc.) prior to cultivation for a further $72 \mathrm{~h}$.

For rescue experiments, HUVECs $\left(2 \times 10^{5}\right)$ from the NC and CLDN2 overexpression groups were seeded in 24-well plates containing RPMI-1640 with $10 \%$ FBS. When $60 \%$ confluence 
was reached, HUVECs were transfected with miR-NC and miR-331 mimics as described above. After cultivation for $48 \mathrm{~h}$, cells were collected for further analysis.

CCK- 8 assay. HUVECs $\left(2 \times 10^{3}\right.$ cells/well) in the NC, CLDN2 mimics and rescue (miR-331 upregulation) groups were seeded in 96-well plates. At 0, 24, 48 and $72 \mathrm{~h}, 20 \mu \mathrm{l} \mathrm{CCK-8} \mathrm{(5} \mathrm{g/l)}$ was added to the cells, which were then incubated at $37^{\circ} \mathrm{C}$ for $30 \mathrm{~min}$. Then, the absorbance was measured at $490 \mathrm{~nm}$ and the results were used to plot cell proliferation curves. Each group was tested in three replicate wells and the mean values were determined.

Flow cytometry. At $24 \mathrm{~h}$ after transfection, cells $\left(1 \times 10^{6}\right)$ from each group were washed with pre-cooled PBS (2X) and subjected to flow cytometry using the Cell Cycle Assay kit (BD Biosciences) to detect the cell cycle distribution according to the manufacturer's instructions. The data were analyzed using ModFit software (v4.1; Verity Software House, Inc.).

After treatment with serum from healthy subjects or sepsis patients for $24 \mathrm{~h}$, HUVECs $\left(1 \times 10^{6}\right)$ in each group were washed with pre-cooled PBS (2X) and subjected to flow cytometry using the ANXN V-FITC Apoptosis Detection kit I (BD Biosciences) to detect apoptosis according to the manufacturer's instructions. Annexin V-positive cells were early apoptotic, PI-positive cells were necrotic and double-positive cells were late apoptotic (CellQuest v5.1; BD Biosciences).

Transwell assay. In the upper chamber of 24-well Transwell chambers (pore diameter, $8 \mu \mathrm{m}$; Corning Inc.), $200 \mu \mathrm{l}$ serum-free RPMI-1640 containing $2 \times 10^{5}$ HUVECs from each group was added. In addition, $600 \mu 1$ RPMI-1640 with $10 \%$ FBS was added to the lower chamber. After $24 \mathrm{~h}$, the Transwell insert was removed and the cells in the upper chamber were wiped off. After fixing with $4 \%$ formaldehyde at room temperature for $10 \mathrm{~min}$, the membrane was stained using Giemsa staining at room temperature for $2 \mathrm{~min}$ for light microscopic observation of five random fields (magnification, $\mathrm{x} 100)$. The number of migrated cells was calculated.

Western blotting. Cells in each group were trypsinized and collected. Precooled RIPA lysis buffer (600 $\mu \mathrm{l}$; Beyotime Institute of Biotechnology) was added to the samples. After lysis for $30 \mathrm{~min}$ on ice, the mixture was centrifuged at $14,000 \mathrm{x} \mathrm{g}$ for $10 \mathrm{~min}$ at $4^{\circ} \mathrm{C}$. The protein concentration in the supernatant was determined by bicinchoninic acid assay. Protein samples $(20 \mu \mathrm{g})$ were then mixed with 5X SDS loading buffer before denaturation in a boiling water bath for $10 \mathrm{~min}$. Then, samples $(15 \mu \mathrm{g})$ were separated on $10 \%$ SDS-PAGE gels and transferred to polyvinylidene difluoride membranes on ice. Membranes were blocked with $50 \mathrm{~g} / 1$ skimmed milk at room temperature for $1 \mathrm{~h}$ followed by incubation with rabbit anti-human CLDN2 polyclonal primary antibody (1:1,000; cat. no. ab53032; Abcam) and mouse anti-human GAPDH primary antibody (1:4,000; cat. no. AF0006; Beyotime Institute of Biotechnology) at $4^{\circ} \mathrm{C}$ overnight. After washing with PBS containing Tween-20 (1\%) five times at room temperature for $5 \mathrm{~min}$, the membranes were incubated with goat anti-rabbit (cat. no. sc-2004) and goat anti-mouse (cat. no. sc-2005) immunoglobulin $\mathrm{G}$ horseradish peroxidase-conjugated secondary antibodies (1:3,000; Santa Cruz Biotechnology, Inc.) for $1 \mathrm{~h}$ at room temperature before repeating the washing step. Membranes were developed using an enhanced chemiluminescence detection kit (Sigma-Aldrich; Merck KGaA) for imaging. Image lab v3.0 (Bio-Rad Laboratories, Inc.) was used to analyze the signals and the CLDN2/GAPDH ratio was determined.

Bioinformatics. To understand the regulatory mechanism of CLDN2, TargetScan (http://www.targetscan.org) was used to predict miRNA molecules that may regulate CLDN2.

Dual luciferase reporter assay. Based on the bioinformatics results, wild-type (WT) and mutant seed regions of the 3'-UTR of CLDN2 that is predicted to interact with miR-331 were synthesized in vitro utilizing the SpeI and HindIII restriction sites and cloned into the pMIR-REPORT luciferase reporter plasmid (cat. no. D2106; Beyotime Institute of Biotechnology). Plasmids $(0.5 \mu \mathrm{g})$ containing the negative control (NC) for the WT, the WT, the NC for mutant or the mutant 3'-UTR sequences were co-transfected with miR-331 mimics (100 nM; 5'-CTAGGTATGGTCCCAGGGATCC-3'; Sangon Biotech Co., Ltd.) into 293 cells (The Cell Bank of Type Culture Collection of the Chinese Academy of Sciences) using Lipofectamine ${ }^{\mathrm{TM}} 2000$ according to the manufacturer's instructions (Invitrogen; Thermo Fisher Scientific, Inc.). After cultivation for $24 \mathrm{~h}$, cells were lysed and analyzed using the dual luciferase reporter assay kit (Promega Corporation) according to the manufacturer's instructions and the fluorescence intensity was measured using a GloMax 20/20 luminometer (Promega Corporation). Renilla was used as an internal reference.

Statistical analysis. The results were analyzed using SPSS 17.0 (IBM Corp.). The data are expressed as the mean \pm standard deviation $(n \geq 3)$. Comparisons between two groups were performed using Student's t-test. Comparisons of $>2$ groups were performed by one-way ANOVA followed by Student-Newman-Keuls tests. Spearman's correlation analysis was performed to assess correlation. $\mathrm{P}<0.05$ was considered to indicate a statistically significant difference.

\section{Results}

Elevated CLDN2 expression alters the proliferation and cell cycle of peripheral vascular endothelial cells. To determine the expression of CLDN2 and understand how it affects the cellular function of HUVECs, RT-qPCR, Western blotting, CCK-8 and flow cytometry assays were performed. The data showed that CLDN2 mRNA levels in the peripheral blood from patients with sepsis were significantly higher than those in healthy subjects $(\mathrm{P}<0.05$; Fig. 1A). Similarly, CLDN2 protein expression in HUVECs treated with serum from patients with sepsis was significantly increased compared with that in HUVECs treated with serum from healthy subjects $(\mathrm{P}<0.05$; Fig. 1B). The CCK-8 assay showed that the proliferation of HUVECs in the sepsis group was significantly reduced compared with that in the negative control group after 48 and $72 \mathrm{~h}(\mathrm{P}<0.05$; Fig. 1C). Flow cytometry demonstrated that the treatment of HUVECs with serum from patients with sepsis 

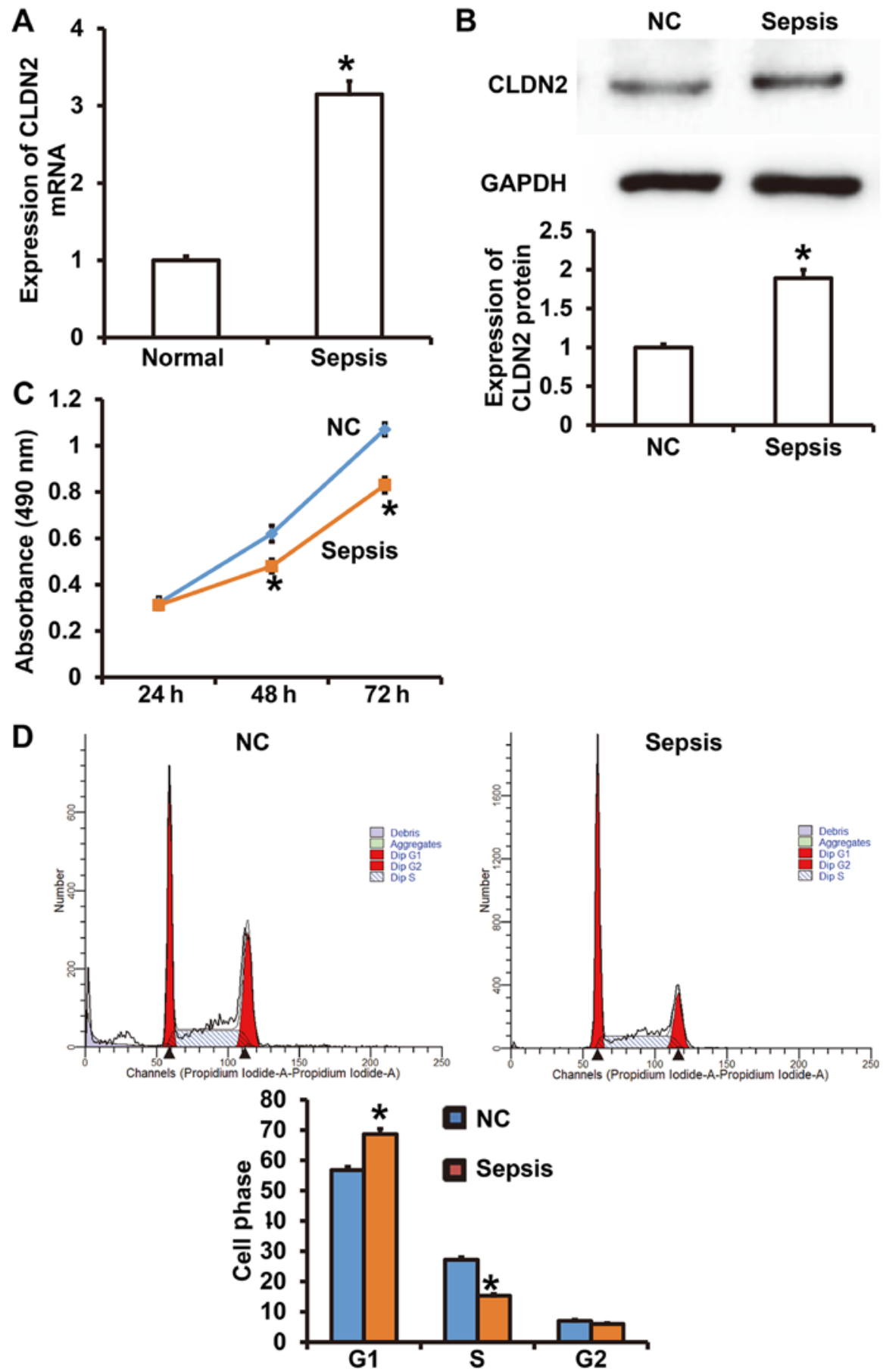

Figure 1. Expression of CLDN2 and its association with sepsis. (A) Expression of CLDN2 mRNA in peripheral blood from patients with sepsis. Reverse transcription-quantitative PCR was used to determine the expression of CLDN2 mRNA "P<0.05 vs. healthy subjects. (B) Expression of CLDN2 protein in HUVECs incubated with serum from healthy subjects or patients with sepsis as determined using Western blotting. (C) Proliferation of HUVECs incubated with serum from healthy subjects or patients with sepsis determined using a Cell Counting Kit- 8 assay. ${ }^{*} \mathrm{P}<0.05$ vs. NC. (D) Cell cycle distributions of HUVECs incubated with serum from healthy subjects or patients with sepsis detected using flow cytometry. "P<0.05 vs. NC. Comparison between two groups was carried out using Student's t-test. CLDN2, claudin-2; NC, negative control, serum from healthy subjects.

decreased transition from the G1 phase to the S phase of the cell cycle compared with that in HUVECS treated with serum from healthy controls $(\mathrm{P}<0.05$; Fig. 1D). The results suggest that elevated CLDN2 expression altered the proliferation and cell cycle of peripheral vascular endothelial cells.

CLDN2 overexpression inhibits the proliferation of HUVECs via mechanisms other than those affecting the cell cycle. To test how CLDN2 overexpression affected the proliferation of
HUVECs, an analysis, including CCK-8 assays was performed. Western blotting demonstrated that the expression of CLDN2 in HUVECs transfected with a CLDN2 overexpression vector was significantly higher than that in HUVECs transfected with the NC vector $(\mathrm{P}<0.05$; Fig. $2 \mathrm{~A})$. The results of the CCK-8 assay showed that the proliferation of HUVECs overexpressing CLDN2 was significantly lower compared with that of the NC group after 48 and $72 \mathrm{~h}(\mathrm{P}<0.05$; Fig. 2B). In addition, flow cytometric analysis showed that the overexpression of CLDN2 

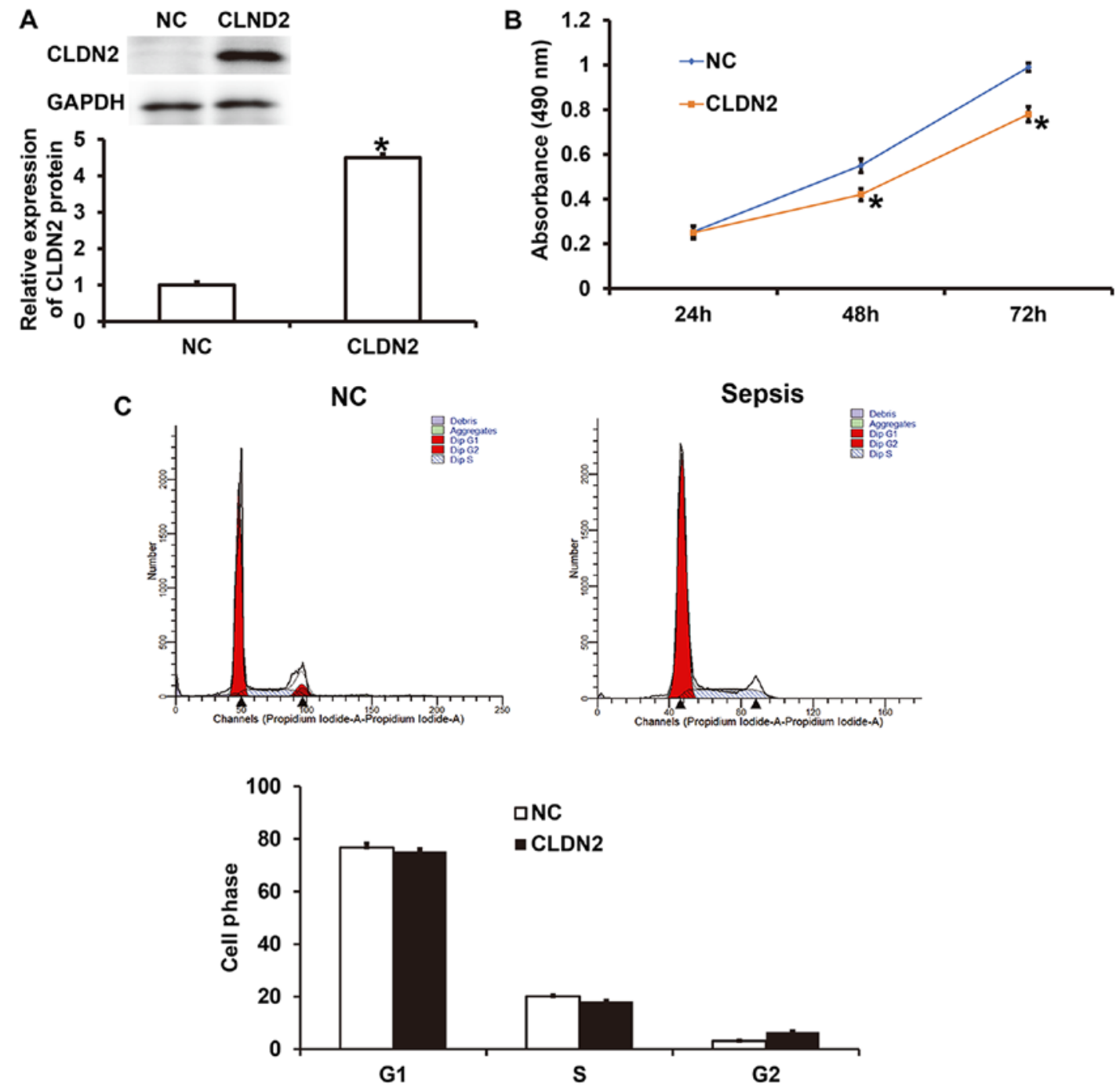

Figure 2. Effect of CLDN2 overexpression on the proliferation and cell cycle of HUVECs. (A) Expression of CLDN2 protein in HUVECs transfected with NC or CLDN2 mRNA as determined using Western blotting. (B) Proliferation of HUVECs transfected with NC or CLDN2 mRNA determined using a Cell Counting Kit-8 assay. (C) Cell cycle of HUVECs transfected with NC or CLDN2 mRNA detected using flow cytometry. ${ }^{*}<0.05$ vs. NC. Comparison between two groups was carried out using Student's t-test. CLDN2, claudin-2; NC, negative control.

did not significantly change the ratio of cells in the G1, S and G2/M phases of the cell cycle compared with those in the NC group ( $\mathrm{P}>0.05$; Fig. $2 \mathrm{C}$ ). These results indicate that CLDN2 overexpression may inhibit the proliferation of HUVECs via mechanisms other than via affecting the cell cycle.

CLDN2 overexpression promotes the apoptosis of HUVECs. To examine whether CLDN2 overexpression affected the apoptosis of HUVECs, flow cytometry was performed. The data showed that number of apoptotic cells was significantly higher in HUVECS overexpressing CLDN2 compared with the $\mathrm{NC}$ group $(\mathrm{P}<0.05$; Fig. 3$)$. The result suggest that CLDN2 overexpression promotes the apoptosis of HUVECs.

CLDN2 overexpression inhibits HUVEC migration. To examine the migration ability of HUVECs, Transwell assays were employed. The data showed that the number of migrated cells was significantly reduced in CLDN2 overexpressing cells compared with the $\mathrm{NC}$ group $(\mathrm{P}<0.05$; Fig. 4). The result indicates that the overexpression of CLDN2 inhibits HUVEC migration.
miR-331 binds to the 3'-UTR of CLDN2 and regulates its expression. TargetScan analysis indicated that miR-133 may bind to the 3'-UTR of CLDN2 (Fig. 5A). To detect the interaction between miR-331 and CLDN2, a dual luciferase reporter assay was performed. Renilla activity in cells co-transfected with agomiR-331 and pMIR-REPORT-WT luciferase reporter plasmids was significantly lower than in the NC group $(\mathrm{P}<0.05$; Fig. 5B). By contrast, the activity of cells co-transfected with agomiR-331 and the pMIR-REPORT-mutant luciferase reporter plasmids was not significantly different from that in the NC group ( $\mathrm{P}>0.05$; Fig. 5B). The results suggest that miR-331 can bind to the 3'-UTR of CLDN2 mRNA and regulate its expression.

Upregulation of miR-331 expression inhibits the expression of $C L D N 2$ and restores the proliferation, apoptosis and migration of HUVECs. To study the association of miR-331 with CLDN2 expression in the peripheral blood of patients with sepsis, a correlation analysis was performed. The data indicate that miR-331 expression was negatively correlated with CLDN2 mRNA expression in 15 patients with sepsis (Fig. 6A). 

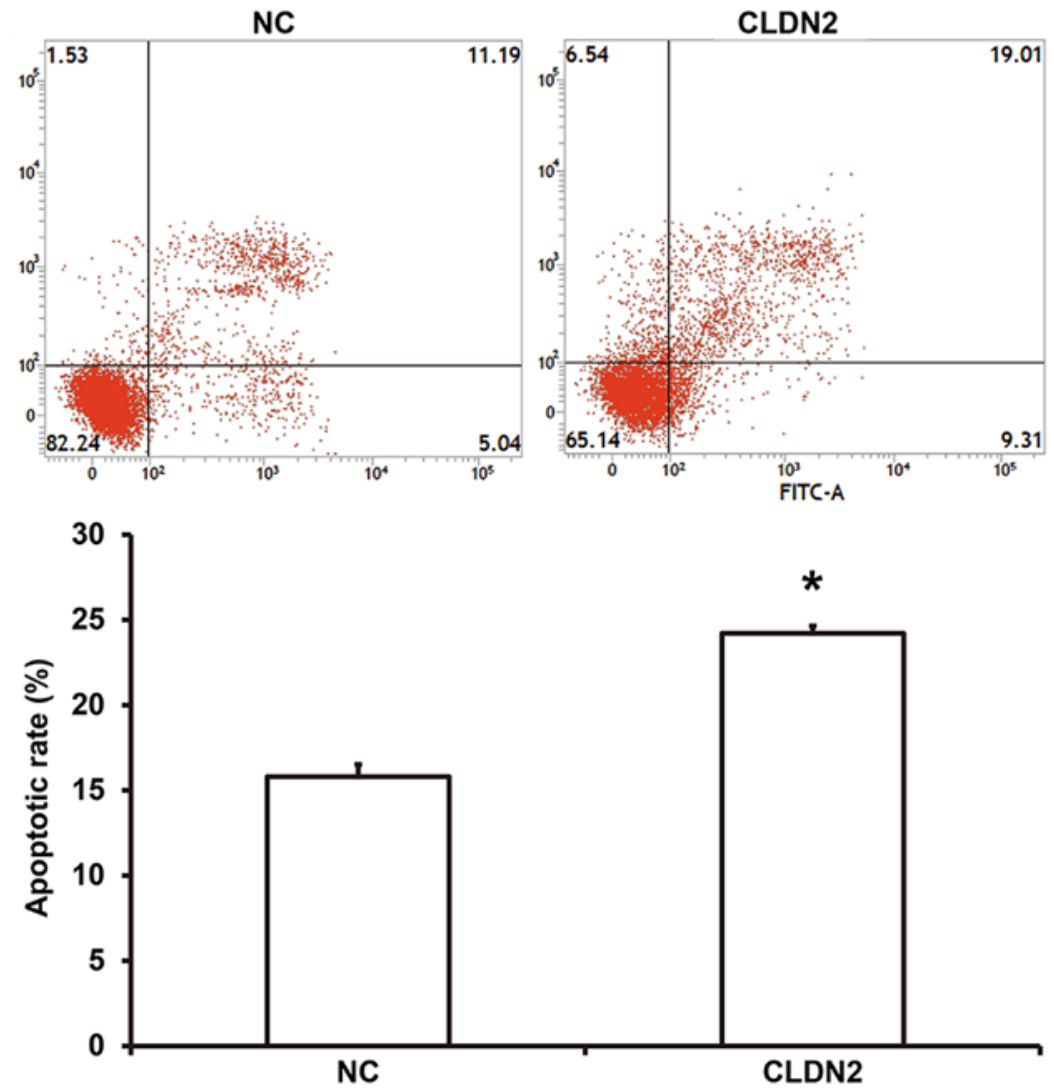

Figure 3. Effect of CLDN2 overexpression on the apoptosis of HUVECs. Flow cytometry was used to detect apoptosis and to calculate the apoptotic rate. "P<0.05 vs. NC. Comparison between two groups was carried out using Student's t-test. CLDN2, claudin-2; NC, negative control.
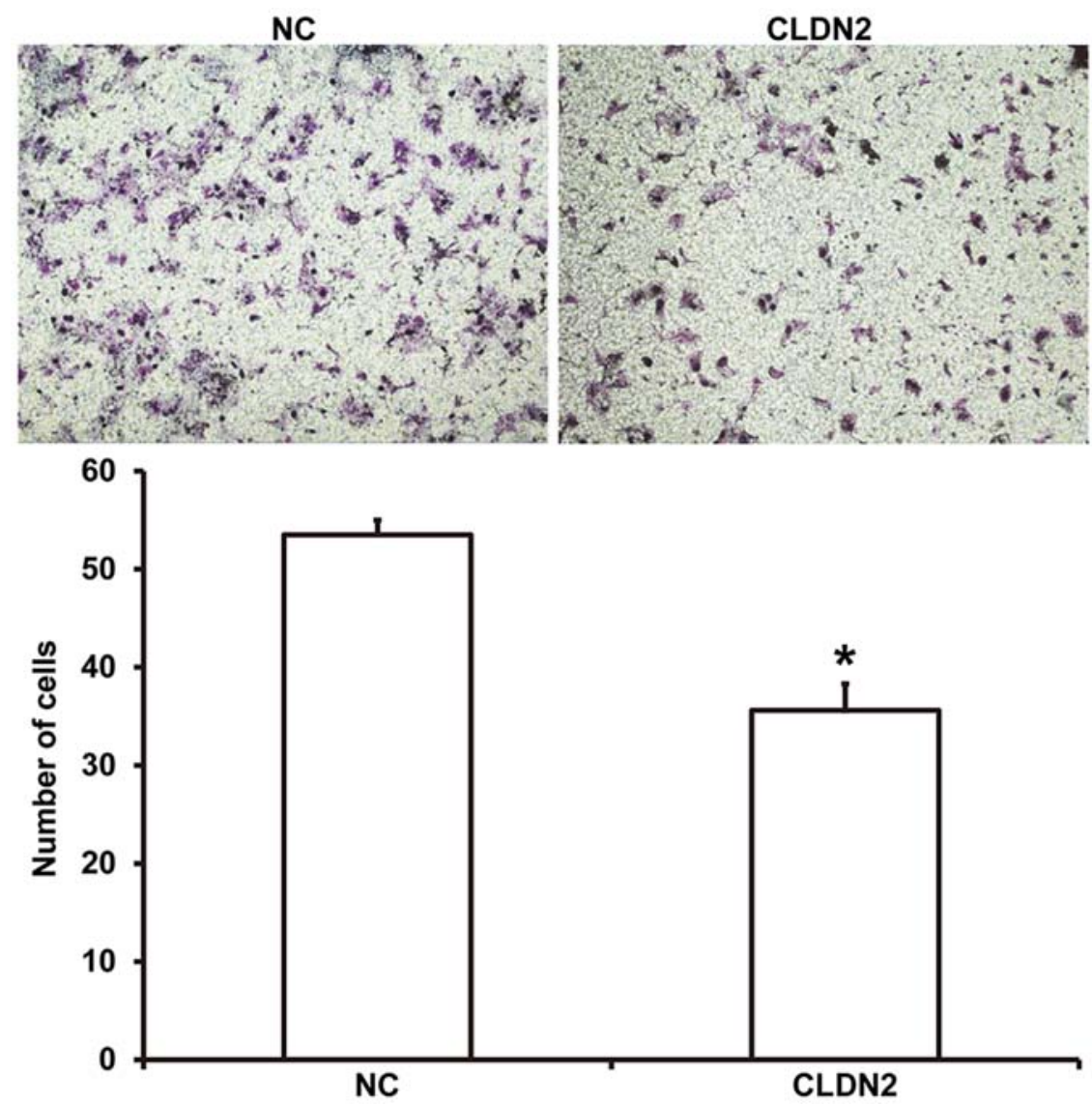

Figure 4. Effect of CLDN2 overexpression on the migration of HUVECs. Transwell assay was used to examine the migration ability of cells. Magnification, $\mathrm{x} 100$. ${ }^{*} \mathrm{P}<0.05$ vs. NC. Comparison between two groups was carried out using Student's t-test. CLDN2, claudin-2; NC, negative control. 
A

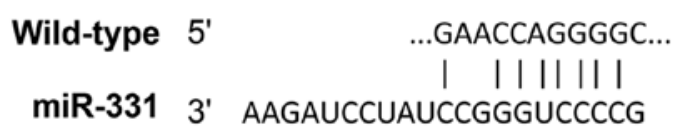

\author{
Mutant $5^{\prime}$ \\ miR-331 3' AAGAUCCUAUCCGGGUCCCCG
}

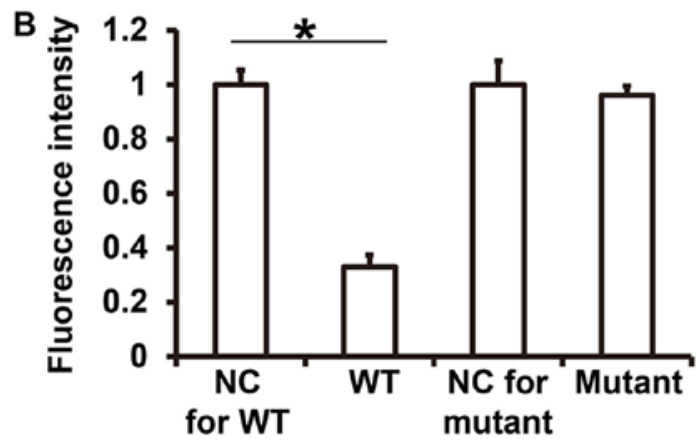

Figure 5. Identification of an interaction between miR-331 and CLDN2 mRNA using a dual luciferase reporter assay. (A) Binding sites of miR-331 with WT or mutant CLDN2 gene. (B) Fluorescence intensity obtained for the WT and mutant 3'-UTR of CLDN mRNA when cotransfected with miR-331 or NC. $P<0.05$ as indicated. Comparison between two groups was carried out using Student's t-test. CLDN2, claudin-2; WT, wild-type; UTR, untranslated region; NC, negative control.

RT-qPCR analysis confirmed that the expression level of miR-331 in HUVECs transfected with miR-331 mimics was significantly higher than that in HUVECs transfected with miR-NC $(\mathrm{P}<0.05$; Fig. 6B). At the cellular level, the CLDN2 protein expression in HUVECs transfected with miR-331 mimics was significantly lower than that in the miR-NC group $(\mathrm{P}<0.05$; Fig. 6C). In the CCK-8 assay, rescue experiments showed that the upregulation of miR-331 significantly increased the absorbance at 48 or $72 \mathrm{~h}$ in HUVECs overexpressing CLDN2 (rescue group) compared with that in HUVECS overexpressing CLDN2 and transfected with miR-NC (CLDN2 group) $(\mathrm{P}<0.05)$, and restored the absorbance to levels similar to those in the NC group ( $\mathrm{P}>0.05$; Fig. 6D). Flow cytometry showed that the upregulation of miR-331 significantly reduced the apoptotic rate in the rescue group compared with that in the CLDN2 group $(\mathrm{P}<0.05)$, and restored apoptosis to a level similar to that in the $\mathrm{NC}$ group $(\mathrm{P}>0.05$; Fig. $6 \mathrm{E})$. In addition, the Transwell assay showed that the upregulation of miR-331 significantly elevated the migration ability of the rescue group compared with that in the CLDN2 group $(\mathrm{P}<0.05)$, and raised it to a level comparable with that of the $\mathrm{NC}$ group ( $P>0.05$; Fig. 6F). The results indicate that upregulation of miR-331 expression inhibits the expression of CLDN2 and restores nearly normal proliferation, apoptosis and migration to HUVECs.

\section{Discussion}

Sepsis is a clinically common complication. Without timely treatment, it often induces systemic inflammatory responses, circulatory failure, systemic organ damage or even death (26). Vascular endothelial cells are natural barriers in the walls of blood vessels, and tight junction proteins can crosslink endothelial cells mechanically, regulate the transport of intracellular and extracellular substances, and maintain the vascular endothelial barrier (27). In the present study, it was discovered that the mRNA of the tight junction protein CLDN2 is upregulated in the peripheral blood of patients with sepsis. In vitro experiments revealed that serum from patients with sepsis promoted the expression of CLDN2 in HUVECs, which inhibited the proliferation and migration of HUVECs, and promoted apoptosis.

CLDN2 is a member of the claudin family. Claudin proteins play important roles in the formation and maintenance of tight junctions among cells (28). Tight junctions divide the epithelial plasma membrane into apical and basal sides, resulting in cell polarity, but have selective permeability to different types of molecules and ions (29). It has been discovered that the abnormal expression of CLDN2 protein is closely associated with cell proliferation, migration, apoptosis and inflammation. For example, the abnormal expression of CLDN2 in proximal renal tubular epithelial cells often leads to apoptosis, necrosis and detachment, which induces the occurrence of acute tubular necrosis (30). The infection of endothelial cells with KSHV downregulates the expression of CLDN2, resulting in impairment of the endothelial cell barrier (31). Disordered CLDN2 expression also serves an important role in tumor cells. For example, Du et al (32) found that Spi-B-induced silencing of the CLDN2 gene promoted the distal metastasis of lung cancer cells in mice. In addition, CLDN2 can be used as an indicator for the prognosis of breast cancer, and higher expression of CLDN2 usually corresponds to worse prognosis (33). In the present study, it was found that the expression of CLDN2 mRNA in the peripheral blood of patients with sepsis was significantly higher than in healthy subjects, and it was hypothesized that CLDN2 may be associated with sepsis and vascular endothelial injury. For HUVECs cultured in the presence of serum from patients with sepsis, the expression of CLDN2 was significantly elevated, the proliferation and migration of the HUVECs were reduced, and apoptosis was increased. Furthermore, upregulation of CLDN2 expression inhibited the proliferation and migration of HUVECs, and promoted their apoptosis. These results also suggest that CLDN2 promotes the damage of HUVECs and may serve an important role in vascular endothelial injury in sepsis.

miR-331 is a type of miRNA that is located at 12q22 (34). In an immortalized lymphoblastoid cell line, miR-331 was shown to be associated with the expression of a variety of cell cycle-associated mRNAs, suggesting that miR-331 may be associated with cell proliferation (35). Also, a study revealed that p53 deficiency induces the expression of miR-331 in mouse and embryo development (36). Furthermore, it has been reported that miR-331-3p is abnormally expressed in a number of tumors, such as gastric and prostate cancer, and is closely associated with the proliferation and migration of tumor cells (37). In the present study, bioinformatics analysis indicated that the 3'-UTR of CLDN2 contains binding sites for miR-331, suggesting that miR-331 may be involved the 


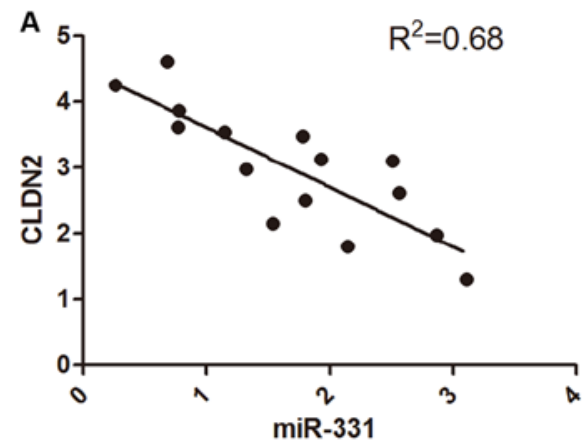

C miR-NC miR-331 mimics
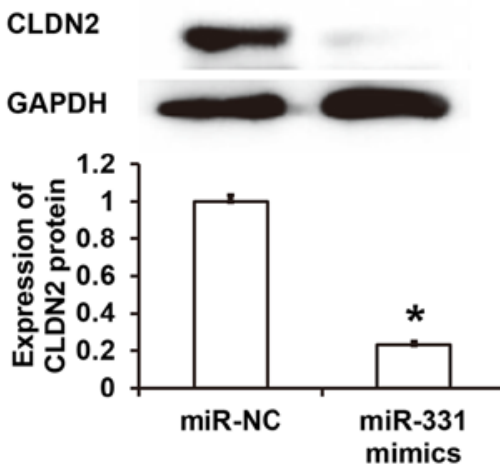

B

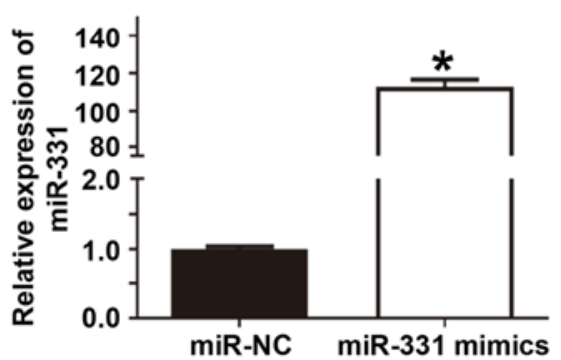

D

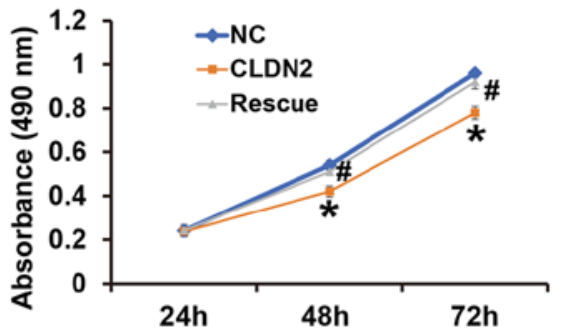

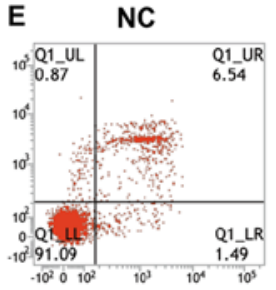

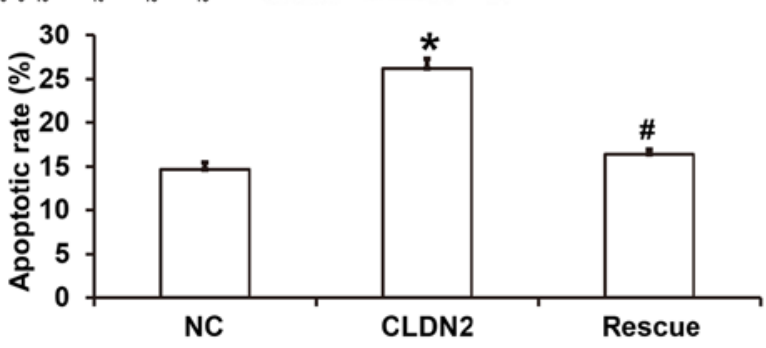

CLDN2
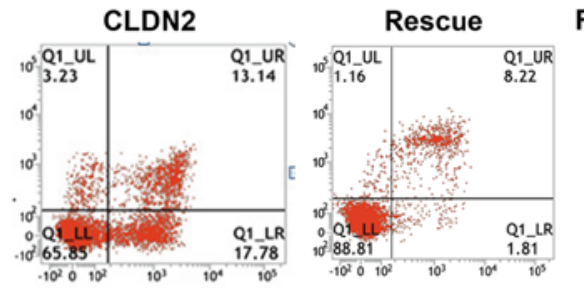

NC

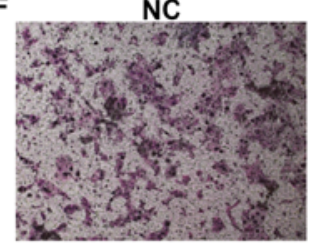

60

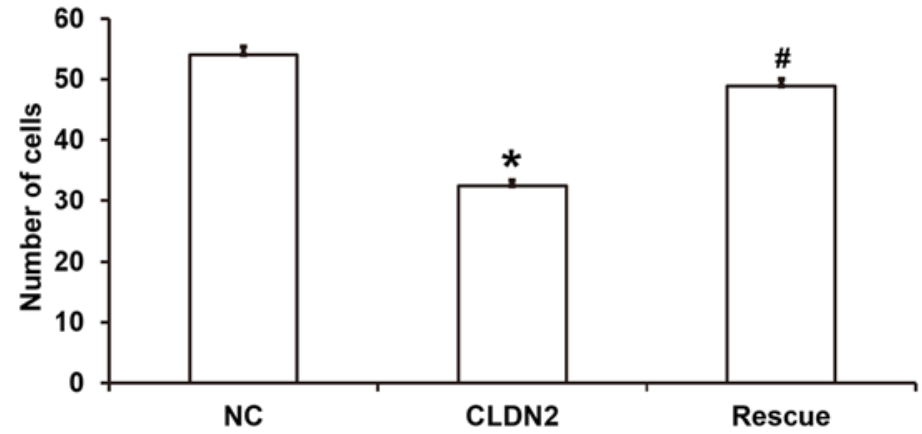

CLDN2
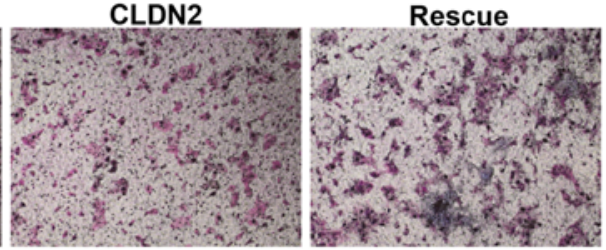

Figure 6. Correlation between miR-331 and CLDN2 and their effect on cellular functions. (A) Correlation between miR-331 expression and CLDN2 expression in peripheral blood from patients with sepsis analyzed using Spearman's correlation analysis. (B) Expression level of miR-331 in HUVECs transfected with miR-NC or miR-331 mimics. Reverse transcription-quantitative PCR was used to determine the expression of miR-331. Student's t-test was used for statistical analysis. "P $<0.05$ vs. miR-NC group. (C) Expression of CLDN2 protein in HUVECs transfected with miR-NC or miR-331 mimics as determined using Western blotting. Student's t-test was used for statistical analysis. "P<0.05 vs. miR-NC group. (D) Proliferation of HUVECs in the NC, CLDN2 and rescue groups. Cells in the $\mathrm{NC}$ group were not transfected, those in CLDN2 group stably expressed CLDN2, and those in the rescue group stably expressed CLDN2 and were transfected with miR-331. A Cell Counting Kit-8 assay was used to determine the proliferation. One-way ANOVA followed by Student-Newman-Keuls tests were used for statistical analysis. ${ }^{*} \mathrm{P}<0.05$ vs. $\mathrm{NC}$ and ${ }^{~} \mathrm{P}<0.05$ vs. CLDN2 at the same time point. No statistically significant difference was observed between the NC and rescue groups. (E) Apoptotic rate of HUVECs in the negative control, CLDN2 and rescue groups as determined by flow cytometry. One-way ANOVA followed by Student-Newman-Keuls tests were used for statistical analysis. " $\mathrm{P}<0.05$ vs. NC and ${ }^{\text {"P }}<0.05$ vs. CLDN2. No statistically significant difference was observed between the NC and rescue groups. (F) Migration ability of HUVECs in the NC, CLDN2 and rescue groups as determined by Transwell assay. Magnification, x100. ${ }^{*} \mathrm{P}<0.05$ vs. NC and ${ }^{\#} \mathrm{P}<0.05$ vs. CLDN2. One-way ANOVA followed by Student-Newman-Keuls tests were used for statistical analysis. No statistically significant difference was observed between the NC and rescue groups. CLDN2, claudin-2; WT, wild-type; UTR, untranslated region; NC, negative control.

regulation of CLDN2 expression. A dual luciferase reporter assay confirmed that miR-331 directly binds with the 3'-UTR of CLDN2 in vitro, suggesting that CLDN2 is a direct target gene of miR-331. Moreover, in the present study, upregulation of miR-331 decreased the expression of CLDN2 in HUVECs, and miR-331 overexpression reversed the effect of CLDN2 on the cellular functions of HUVECs. These results indicate that CLDN2 expression in HUVECs is regulated by miR-331. However, more evidence is required to confirm the direct regulatory relationship between miR-331 and CLDN2. At present, the most convincing experiment the present authors were able to perform to demonstrate the direct interaction 
between miR-331 and CLDN2 is dual luciferase report assay. Future studies are planned to obtain improved experimental data, such as by the use of in situ hybridization or RT-qPCR. A further limitation of the present study is that the biological functions of CLDN2 were only investigated in vitro. The functions of CLDN2 in endothelial cell injury in vivo remain to be determined in future studies.

In conclusion, the present study demonstrated that the expression of CLDN2 is upregulated in the peripheral blood of patients with sepsis. In addition, CLDN2 upregulation is directly associated with miR-331 downregulation, leading to the inhibition of proliferation and migration, and the promotion of apoptosis of vascular endothelial cells.

\section{Acknowledgements}

The authors would like to thank Dr Zhigang Zhang, the Director of Linyi Central Hospital, for his supervision of the study, and Dr Wenhong Peng, the Director of Department of Critical Care Medicine, Linyi Central Hospital, for advice regarding the writing up of the article.

\section{Funding}

No funding was received.

\section{Availability of data and materials}

The datasets used and/or analysed during the current study are available from the corresponding author on reasonable request.

\section{Authors' contributions}

LK and JL contributed to the design of the study. LK and PW performed the experiments. LK and JL analysed the data, interpreted the results and prepared the manuscript. All authors read and approved the final manuscript.

\section{Ethics approval and consent to participate}

All procedures were approved by the Ethics Committee of Linyi Central Hospital. Written informed consent was obtained from all patients.

\section{Patient consent for publication}

Not applicable.

\section{Competing interests}

The authors declare that they have no competing interests.

\section{References}

1. Rhee $\mathrm{C}$ and Klompas M: Sepsis trends: Increasing incidence and decreasing mortality, or changing denominator? J Thorac Dis 12 (Suppl 1): S89-S100, 2020.

2. Kuchler L, Sha LK, Giegerich AK, Knape T, Angioni C, Ferreirós N, Schmidt MV, Weigert A, Brüne B and von Knethen A: Elevated intrathymic sphingosine-1-phosphate promotes thymus involution during sepsis. Mol Immunol 90: 255-263, 2017.
3. Chao $\mathrm{CH}$, Chen HR, Chuang YC and Yeh TM: Macrophage migration inhibitory factor-induced autophagy contributes to thrombin-triggered endothelial hyperpermeability in sepsis. Shock 50: 103-111, 2018.

4. Bobelytė O, Gailiūtė I, Zubka V and Žilinskaitè V: Sepsis epidemiology and outcome in the paediatric intensive care unit of Vilnius University Children's Hospital. Acta Med Litu 24: 113-120, 2017.

5. Malik IA, Cardenas-Turanzas M, Gaeta S, Borthakur G, Price K, Cortes J and Nates JL: Sepsis and acute myeloid leukemia: A population-level study of comparative outcomes of patients discharged from Texas hospitals. Clin Lymphoma Myeloma Leuk 17: e27-e32, 2017.

6. Dombrovskiy VY, Martin AA, Sunderram J and Paz HL: Rapid increase in hospitalization and mortality rates for severe sepsis in the United States: A trend analysis from 1993 to 2003. Crit Care Med 35: 1244-1250, 2007.

7. Raju R: Immune and metabolic alterations following trauma and sepsis - An overview. Biochim Biophys Acta Mol Basis Dis 1863: 2523-2525, 2017

8. Katayama S, Nunomiya S, Koyama K, Wada M, Koinuma T, Goto Y, Tonai K and Shima J: Markers of acute kidney injury in patients with sepsis: The role of soluble thrombomodulin. Crit Care 21: 229, 2017.

9. Sergi C, Shen F, Lim DW, Liu W, Zhang M, Chiu B, Anand V and Sun Z: Cardiovascular dysfunction in sepsis at the dawn of emerging mediators. Biomed Pharmacother 95: 153-160, 2017.

10. Schaalan M and Mohamed W: Predictive ability of circulating osteoprotegerin as a novel biomarker for early detection of acute kidney injury induced by sepsis. Eur Cytokine Netw 28: 52-62, 2017.

11. Rieg S, Bechet L, Naujoks K, Hromek J, Lange B, Juzek-Küpper MF, Stete K, Müller MC, Jost I, Kern WV, et al: A single-center prospective cohort study on postsplenectomy sepsis and its prevention. Open Forum Infect Dis 7: ofaa050, 2020.

12. Pluskota E, Bledzka KM, Bialkowska K, Szpak D, Soloviev DA, Jones SV, Verbovetskiy D, Plow EF, et al: Kindlin-2 interacts with endothelial adherens junctions to support vascular barrier integrity. J Physiol 595: 6443-6462, 2017.

13. Sun K, Lei Y, Wang R, Wu Z and Wu G: Cinnamicaldehyde regulates the expression of tight junction proteins and amino acid transporters in intestinal porcine epithelial cells. J Anim Sci Biotechnol 8: 66, 2017.

14. Thuringer D, Solary E and Garrido C: The microvascular gap junction channel: A route to deliver MicroRNAs for neurological disease treatment. Front Mol Neurosci 10: 246, 2017.

15. Li X, Song G, Zhao Y, Zhao F, Liu C, Liu D, Li Q and Cui Z: Claudin $7 \mathrm{~b}$ is required for the formation and function of inner ear in zebrafish. J Cell Physiol 233: 3195-3206, 2018.

16. Kolosov D, Bui P, Wilkie MP and Kelly SP: Claudins of sea lamprey (Petromyzon marinus) - organ-specific expression and transcriptional responses to water of varying ion content. J Fish Biol 96: 768-781, 2020.

17. Taylor NA, Vick SC, Iglesia MD, Brickey WJ, Midkiff BR, McKinnon KP, Reisdorf S, Anders CK, Carey LA, Parker JS, et al: Treg depletion potentiates checkpoint inhibition in claudin-low breast cancer. J Clin Invest 127: 3472-3483, 2017.

18. Chung YH, Li SC, Kao YH, Luo HL, Cheng YT, Lin PR, Tai MH and Chiang PH: MiR-30a-5p inhibits epithelial-to-mesenchymal transition and upregulates expression of tight junction protein claudin-5 in human upper tract urothelial carcinoma cells. Int J Mol Sci 18: 18, 2017.

19. Ahmad R, Kumar B, Chen Z, Chen X, Müller D, Lele SM, Washington MK, Batra SK, Dhawan P and Singh AB: Loss of claudin-3 expression induces IL6/gp130/Stat3 signaling to promote colon cancer malignancy by hyperactivating $\mathrm{Wnt} / \beta$-catenin signaling. Oncogene 36: 6592-6604, 2017.

20. Hashimoto Y, Shirakura K, Okada Y, Takeda H,Endo K, Tamura M, Watari A, Sadamura Y, Sawasaki T, Doi T, et al: Claudin-5-binders enhance permeation of solutes across the blood-brain barrier in a mammalian model. J Pharmacol Exp Ther 363: 275-283, 2017.

21. Tesfaye D, Gebremedhn S, Salilew-Wondim D, Hailay T, Hoelker M, Grosse-Brinkhaus C and Schellander K: MicroRNAs: tiny molecules with significant role in mammalian follicular and oocyte development. Reproduction 155: R121-R135, 2018.

22. Song L, Li D, Li X, Ma L, Bai X, Wen Z, Zhang X, Chen D and Peng L: Exposure to PM2.5 induces aberrant activation of NF- $\kappa B$ in human airway epithelial cells by downregulating miR-331 expression. Environ Toxicol Pharmacol 50: 192-199, 2017.

23. Livak KJ and Schmittgen TD: Analysis of relative gene expression data using real-time quantitative PCR and the $2(-\Delta \Delta C(T)) \mu$ ethod. Methods 25: 402-408, 2001. 
24. Mao FY, Kong H,Zhao YL, Peng LS, Chen W, Zhang JY, Cheng $\mathrm{P}$ Wang TT, LV YP, Teng YS, et al: Increased tumor-infiltrating CD45RA-CCR7- regulatory T-cell subset with immunosuppressive properties foster gastric cancer progress. Cell Death Dis 8: e3002, 2017.

25. Peng LS, Zhang JY, Teng YS, Zhao YL, Wang TT, Mao FY, Lv YP, Cheng P, Li WH, Chen N, et al: Tumor-associated monocytes/macrophages impair NK-cell function via TGF $\beta 1$ in human gastric cancer. Cancer Immunol Res 5: 248-256, 2017.

26. Kam HJ and Kim HY: Learning representations for the early detection of sepsis with deep neural networks. Comput Biol Med 89: 248-255, 2017.

27. Tham CL, Hazeera Harith H, Wai Lam K, Joong Chong Y, Singh Cheema M, Roslan Sulaiman M, Hj Lajis $\mathrm{N}$ and Ahmad Israf D: The synthetic curcuminoid BHMC restores endotoxin-stimulated HUVEC dysfunction: Specific disruption on enzymatic activity of p38 MAPK. Eur J Pharmacol 749: 1-11, 2015.

28. Sumitomo T: Streptococcus pyogenes translocates across an epithelial barrier. Nippon Saikingaku Zasshi 72: 213-218, 2017 (In Japanese).

29. Rosas-Hernandez H, Cuevas E, Escudero-Lourdes C, Lantz SM, Gomez-Crisostomo NP, Sturdivant NM, Balachandran K, Imam SZ, Slikker W Jr, Paule MG, et al: Characterization of Biaxial stretch as an in vitro model of traumatic brain injury to the blood-brain barrier. Mol Neurobiol 55: 258-266, 2018.

30. Fujii N, Matsuo Y, Matsunaga T, Endo S, Sakai H, Yamaguchi M, Yamazaki Y, Sugatani J and Ikari A: Hypotonic stress-induced down-regulation of claudin-1 and - 2 mediated by dephosphorylation and clathrin-dependent endocytosis in renal tubular epithelial cells. J Biol Chem 291: 24787-24799, 2016.

31. Tan X, Li D, Wang X, Zeng Y, Yan Y and Yang L: Claudin-2 downregulation by KSHV infection is involved in the regulation of endothelial barrier function. J Cutan Pathol 41: 630-639, 2014.
32. Du W, Xu X, Niu Q, Zhang X, Wei Y, Wang Z, Zhang W, Yan J, $\mathrm{Ru}$ Y, Fu Z, et al: Spi-B-mediated silencing of claudin-2 promotes early dissemination of lung cancer cells from primary tumors. Cancer Res 77: 4809-4822, 2017.

33. Ma F, Ding X, Fan Y, Ying J, Zheng S, Lu N and Xu B: A CLDN1-negative phenotype predicts poor prognosis in triple-negative breast cancer. PLoS One 9: e112765, 2014.

34. Sierzega M, Kaczor M, Kolodziejczyk P, Kulig J, Sanak M and Richter P: Evaluation of serum microRNA biomarkers for gastric cancer based on blood and tissue pools profiling: The importance of miR-21 and miR-331. Br J Cancer 117: 266-273, 2017.

35. Butrym A, Rybka J, Baczyńska D, Tukiendorf A, Kuliczkowski K and Mazur G: Expression of microRNA-331 can be used as a predictor for response to therapy and survival in acute myeloid leukemia patients. Biomarkers Med 9: 453-460, 2015.

36. Hosako H, Martin GS, Barrier M, Chen YA, Ivanov IV and Mirkes PE: Gene and microRNA expression in p53-deficient day 8.5 mouse embryos. Birth Defects Res A Clin Mol Teratol 85: 546-555, 2009

37. Cao Y, Chen J, Wang D, Peng H, Tan X, Xiong D, Huang A and Tang H: Upregulated in hepatitis B virus-associated hepatocellular carcinoma cells, miR-331-3p promotes proliferation of hepatocellular carcinoma cells by targeting ING5. Oncotarget 6 : 38093-38106, 2015.

This work is licensed under a Creative Commons Attribution-NonCommercial-NoDerivatives 4.0 International (CC BY-NC-ND 4.0) License. 